\title{
TRANSVERSALITIES FOR LAGRANGE SINGULARITIES OF ISOTROPIC MAPPINGS OF CORANK ONE
}

\author{
GOO ISHIKAWA \\ Department of Mathematics, Hokkaido University \\ Sapporo 060, Japan \\ E-mail: ishikawa@math.hokudai.ac.jp \\ and \\ Department of Pure Mathematics, University of Liverpool, \\ P.O. Box 147, Liverpool L69 3BX, England, U.K. \\ E-mail: ishikawa@liverpool.ac.uk
}

0. Introduction. The Lagrange singularity theory connects the Lagrange classification of Lagrange immersions with the classification of families of functions, that is, generating families. (The theory of Hörmander, Arnol'd, Zakalyukin, ..., [AGV]). Though there exist detailed studies on singular Lagrange varieties (e.g. [A1], [A2], [DP], [G1], [G2], [J], $[\mathrm{Z}]$ ), the nice connection between Lagrange singularities and generating families seems to break, in particular, when we try to classify generic Lagrange non-immersions or generic isotropic mappings. Our attempt is then to study singularities of isotropic mappings in the framework of singularity theory of differential mappings (Thom-Mather theory: e.g. [T], [M2]), and to classify their generic singularities under the Lagrange equivalence.

In the singularity theory, the transversality theorem is a powerful tool to grasp generic conditions for differentiable mappings, with respect to partial derivatives (see, for instance, [M1], [M2]). In general, if we fix a space of mappings, a generic condition on a mapping should be described by the transversality of the jet section of the mapping to a stratification naturally defined in a jet space of sufficiently higher order. Then, "the transversality theorem" claims the density of the subspace of mappings satisfying the transversality condition, relatively to an appropriate topology on the space of mappings. As a rule, the validity of "the theorem", however, depends on the space of mappings.

Then, the purpose of this paper is to formulate explicitly and to prove the transversality theorem for the space of isotropic mappings of corank at most one, endowed with

1991 Mathematics Subject Classification: 58C27, 58F05.

The paper is in final form and no version of it will be published elsewhere. 
the Whitney $C^{\infty}$ topology. The corank condition is necessary at least to assure the corresponding jet spaces are non-singular.

In what follows, all manifolds, mappings and differential forms are assumed to be of class $C^{\infty}$.

Isotropic mappings arise naturally in symplectic geometry: Let $X$ be a manifold of dimension $n$, and $M$ a symplectic manifold of dimension $2 n$ with the symplectic form $\omega$. We call a mapping $\phi: X \rightarrow M$ isotropic if the pull-back $\phi^{*} \omega$ is equal to zero. An isotropic immersion is called a Lagrange immersion (cf. [W]), and therefore an isotropic mapping is regarded as a Lagrange immersion with singularities: A point $x \in X$ is a singular point of $\phi$ if the the rank of tangential mapping $\left(\phi_{x}\right)_{*}: T_{x} X \rightarrow T_{\phi(x)} M$ is less than $n$. We call the dimension of the kernel of $\left(\phi_{x}\right)_{*}$, the corank of the germ $\phi_{x}$. By the corank of $\phi$, we mean the supremum of the corank of $\phi_{x}, x \in X$.

Let $J_{I}^{k}(X, M)$ stand for the set of $k$-jets $j^{k} \phi(x)$ of isotropic map-germs $\phi: X, x \rightarrow$ $M, \phi(x), x \in X$. Then, in general, $J_{I}^{k}(X, M) \subset J^{k}(X, M)$ is not a submanifold. We denote by $R^{k}(X, M)$ the set of $k$-jets of isotropic map-germs of corank at most one. Then we see that $R^{k}(X, M)$ is a submanifold of $J^{k}(X, M)$, and moreover the natural projection $\pi_{k, \ell}: R^{k}(X, M) \rightarrow R^{\ell}(X, M)$ is a submersion for $\ell \leq k$ (Corollary 3.3 ).

We denote by $C_{I}^{\infty}(X, M)^{1}$ the set of isotropic mappings $\phi: X \rightarrow M$ with corank $\phi \leq 1$, endowed with the Whitney $C^{\infty}$ topology. For each $\phi \in C_{I}^{\infty}(X, M)^{1}$, we consider the jet extension $j^{k} \phi: X \rightarrow R^{k}(X, M)$.

This paper is devoted to proving the following transversality theorem:

THEOREM 0.1. Let $X$ be an $n$-manifold, $M$ a symplectic $2 n$-manifold, $k$ a non-negative integer and $U$ a locally finite family of submanifolds of $R^{k}(X, M)$. Then the subspace $T_{U}$ of $C_{I}^{\infty}(X, M)^{1}$, consisting of mappings $\phi$ such that the $k$-jet extension $j^{k} \phi$ is transverse to all elements of $U$, is dense in $C_{I}^{\infty}(X, M)^{1}$.

To apply Theorem 0.1 to the singularity theory of isotropic mappings, we need to recall several fundamental notions.

Two isotropic map-germs $\phi: X, x \rightarrow(M, \omega)$ and $\phi^{\prime}: X^{\prime}, x^{\prime} \rightarrow\left(M^{\prime}, \omega^{\prime}\right)$ are called symplectically equivalent if there exist a diffeomorphism-germ $\sigma: X, x \rightarrow X^{\prime}, x^{\prime}$ and a symplectic diffeomorphism-germ $\tau: M, \phi(x) \rightarrow M^{\prime}, \phi^{\prime}\left(x^{\prime}\right), \tau^{*} \omega^{\prime}=\omega$, such that $\tau \circ \phi=$ $\phi^{\prime} \circ \sigma$. Remark that any Lagrange immersion-germs are symplectically equivalent to each other.

In Lagrange singularity theory, it is also natural to consider a symplectic manifold with a Lagrange fibration. The typical example is the cotangent bundle $T^{*} Q$ over an $n$-manifold $Q$ with the symplectic form $\omega=d \theta$, where $\theta$ is the canonical (Liouville) form on $T^{*} Q$, and with the Lagrange fibration $\pi: T^{*} Q \rightarrow Q$.

For an isotropic mapping $\phi: X \rightarrow T^{*} Q$, a Lagrange singular point $x \in X$ of $\phi$ is a point $x$ with corank $(\pi \circ \phi)_{x}<n$. Here we call corank $(\pi \circ \phi)_{x}$, L-corank of $\phi_{x}$. Then it is clear that corank $\phi_{x} \leq \mathrm{L}$-corank $\phi_{x}$, and therefore, a singular point of $\phi$ is necessarily a Lagrange singular point.

Our concern is to give a fundamental tool for classifying Lagrange singularities under Lagrange equivalence: Two isotropic map-germs $\phi: X, x \rightarrow T^{*} Q$ and $\phi^{\prime}: X^{\prime}, x^{\prime} \rightarrow$ $T^{*} Q^{\prime}$ are called Lagrange equivalent if there exist a diffeomorphism-germ $\sigma: X, x \rightarrow$ 
$X^{\prime}, x^{\prime}$ and a symplectic diffeomorphism-germ $\tau: T^{*} Q, \phi(x) \rightarrow T^{*} Q^{\prime}, \phi^{\prime}\left(x^{\prime}\right)$, covering a diffeomorphism-germ $Q \rightarrow Q^{\prime}$ such that $\tau \circ \phi=\phi^{\prime} \circ \sigma$ (see [AGV]).

By Darboux's theorem, any isotropic map-germ $X, x \rightarrow M$ (resp. $X, x \rightarrow T^{*} Q$ ) is symplectically (resp. Lagrange) equivalent to an isotropic map-germ $\mathbf{R}^{n}, 0 \rightarrow T^{*} \mathbf{R}^{n}, 0$, where the symplectic form on $T^{*} \mathbf{R}^{n}$ is given by $\omega=d\left(\sum_{i=1}^{n} p_{i} d q_{i}\right)$.

Denote by $I(n)$ the set of isotropic map-germs $\mathbf{R}^{n}, 0 \rightarrow T^{*} \mathbf{R}^{n}, 0$. Further denote by $S^{k}(n)$ (resp. $\left.L^{k}(n)\right)$ the group of $k$-jets of symplectic equivalence $\{(\sigma, \tau)\}$ (resp. Lagrange equivalence) on $I(n)$. We set $R^{k}(n)=\left\{j^{k} \phi(0) \mid \phi \in I(n)\right.$, corank $\left.\phi \leq 1\right\}$. Then $R^{k}(n)$ is a $S^{k}(n)$-invariant submanifold of the usual jet space $J^{k}(n, 2 n)$, (see Proposition 3.2).

Though the following modified form of the transversality theorem is in fact a special case of Theorem 0.1, it is more useful in the classification problem of generic singularities of isotropic mappings under the symplectic or the Lagrange equivalence.

Corollary 0.2. We fix non-negative integers $n$ and $k$. Let $U$ be a stratification of $R^{k}(n)$. Assume $U$ is invariant under $S^{k}(n)\left(\right.$ resp. $\left.L^{k}(n)\right)$. We denote by $\tilde{U}$ the naturally obtained stratification of $R^{k}(X, M)$ (resp. $\left.R^{k}\left(X, T^{*} Q\right)\right)$, for an $n$-manifold $X$ and a symplectic $2 n$-manifold $M$ (resp. an n-manifold $Q$ ). Then, the subspace $T_{U} \subset C_{I}^{\infty}(X, M)^{1}$ (resp. $\left.C_{I}^{\infty}\left(X, T^{*} Q\right)^{1}\right)$ of isotropic mappings $\phi: X \rightarrow M\left(\right.$ resp. $\left.\phi: X \rightarrow T^{*} Q\right)$ of corank at most one such that the $k$-jet extension $j^{k} \phi: X \rightarrow R^{k}(X, M)\left(\right.$ resp. $\left.R^{k}\left(X, T^{*} Q\right)\right)$ is transverse to $\tilde{U}$, is dense, with respect to the Whitney $C^{\infty}$ topology.

Theorem 0.1 (resp. Corollary 0.2) implies the transversality theorem for Lagrange immersions, which is an implicit base of the generic classification of Lagrange singularities of Lagrange immersions [AGV].

As another application of Theorem 0.1 or Corollary 0.2 , we see that, if $n<4$, then generic isotropic map-germs $\phi: \mathbf{R}^{n}, 0 \rightarrow T^{*} \mathbf{R}^{n}, 0$ of corank one are of L-corank (= corank $\pi \circ \phi$ ) one (Corollary 3.6).

Moreover we see that, in fact, there exists a generic isotropic map-germ $\phi: \mathbf{R}^{4}, 0 \rightarrow$ $T^{*} \mathbf{R}^{4}, 0$ of corank one and of L-corank 2 (Proposition 3.9). This type of singularities is regarded as a degeneration of the Lagrange singularities of type $D_{4}^{ \pm}$of Lagrange immersions. However, we need other techniques to obtain concrete normal forms of the singularities of this type.

In $\S 1$, we study the structure of isotropic mappings under both symplectic and Lagrange equivalences. The results obtained are used to the proof of Theorem 0.1, as well as they are interesting in theirselves.

The notion of jet is essential for the usual singularity theory. We give in $\S 2$ some simple descriptions of jet-spaces for the counterpart of the singularity theory of isotropic mappings.

We prove Theorems $0.1 \mathrm{in} \S 3$, using the results of $\S \S 1$ and 2 . We also give a simple consequence from Theorem 0.1 and Corollary 0.2.

For brevity, we denote by $E_{n}$ the $\mathbf{R}$-algebra of map-germs $\mathbf{R}^{n}, 0 \rightarrow \mathbf{R}$, and by $m_{n}$ the unique maximal ideal consisting of map-germs $\mathbf{R}^{n}, 0 \rightarrow \mathbf{R}, 0$.

The author would like to thank S. Janeczko for kindly giving him the opportunity to write down this paper, and S. Izumiya, T. Morimoto and T. Ohmoto for valuable com- 
ments. This work has been completed during the author's stay at the University of Liverpool; he would like to express his gratitude especially to J. W. Bruce and C. T. C. Wall for their hospitality.

1. Isotropic mappings. Let $\phi_{0} \in I(n)$ and $\phi_{s}\left(s \in \mathbf{R}^{\ell}, 0\right)$ be an isotropic deformation of $\phi_{0}$. By definition, we are assuming that $\Phi=\left(\phi_{s}, s\right): \mathbf{R}^{n} \times \mathbf{R}^{\ell}, 0 \rightarrow T^{*} \mathbf{R}^{n} \times \mathbf{R}^{\ell}, 0$ is a $C^{\infty}$ map-germ, and that $\phi_{s}^{*} \omega=0$, for all $s \in \mathbf{R}^{\ell}, 0$, and, of course, when $s=0$, $\phi_{s}$ is equal to the given $\phi_{0}$.

Let $x=\left(x_{1}, \ldots, x_{n}\right)$ denote the coordinate system of $\mathbf{R}^{n}, q=\left(q_{1}, \ldots, q_{n}\right)$ the coordinate system of the base space of $T^{*} \mathbf{R}^{n}$, and $(p ; q)=\left(p_{1}, \ldots, p_{n} ; q\right)$ the associated canonical coordinate with the Liouville form $\theta=\sum_{i=1}^{n} p_{i} d q_{i}$. Then $\omega=d \theta$.

Since $\phi_{s}^{*} \omega=d_{x} \phi_{s}^{*}\left(\sum_{i=1}^{n} p_{i} d q_{i}\right)=0$, there exists a family of (generating) functions $e_{s}$ uniquely up to the addition of a function of $s$ such that $d_{x} e_{s}=\phi_{s}^{*}\left(\sum_{i=1}^{n} p_{i} d q_{i}\right)$, where $d_{x}$ means the exterior derivative with respect to $x$. Then we have

$$
d e_{s}=\sum_{i=1}^{n} p_{i} \circ \phi_{s} d\left(q_{i} \circ \phi_{s}\right)+\sum_{j=1}^{\ell}\left(r_{j}\right)_{s} d s_{j},
$$

for some function-germs $\left(r_{j}\right)_{s} \in E_{n}$. If we set

$$
\tilde{\Phi}=\left(\phi_{s} ; r_{s}, s\right): \mathbf{R}^{n} \times \mathbf{R}^{\ell}, 0 \rightarrow T^{*} \mathbf{R}^{n+\ell},
$$

then $\tilde{\Phi}$ is isotropic and it is a lift of $\Phi$ with respect to the projection $\pi: T^{*} \mathbf{R}^{n+\ell} \rightarrow$ $T^{*} \mathbf{R}^{n} \times \mathbf{R}^{\ell}$ defined by $\pi(p, q ; r, s)=(p, q, s)$. As easily verified, any isotropic lifts of $\Phi$ are Lagrange equivalent to $\tilde{\Phi}$. We call $\tilde{\Phi}$ an isotropic unfolding of $\phi_{0}$. Then we have the following fundamental fact:

Proposition 1.1. Let $\phi: X^{n}, x_{0} \rightarrow M^{2 n}, \phi\left(x_{0}\right)$ be an isotropic map-germ of corank $k$. Then $\phi$ is symplectically equivalent to an isotropic unfolding of a $\phi_{0} \in I(k)$ of corank $k$.

P r o of. From symplectic linear algebra, it is easy to see that there exist symplectic coordinates $\left(p_{1}, \ldots, p_{n} ; q_{1}, \ldots, q_{n}\right)$ of $M, \phi\left(x_{0}\right)$ such that $\left(q_{k+1}, \ldots, q_{n}\right) \circ \phi$ is a submersion. Then $\left(x_{k+1}, \ldots, x_{n}\right)=\left(q_{k+1}, \ldots, q_{n}\right) \circ \phi$ is a part of a coordinate system $x=\left(x_{1}, \ldots, x_{n}\right)$ of $\mathbf{R}^{n}, 0$. If we set $s=\left(x_{k+1}, \ldots, x_{n}\right)$, and $\phi_{0}=\left.\left(p_{1}, \ldots, p_{k} ; q_{1}, \ldots, q_{k}\right) \circ \phi\right|_{s=0}$, then $\phi$ is an isotropic unfolding of $\phi_{0}$.

By the same proof as Proposition 1.1, we have

Proposition 1.2. Let $\phi: X^{n}, x_{0} \rightarrow T^{*}\left(Q^{n}\right), \phi\left(x_{0}\right)$ be an isotropic map-germ. Assume the $L$-corank of $\phi(=\operatorname{corank} \pi \circ \phi)$ is equal to $k$. Then $\phi$ is Lagrange equivalent to an isotropic unfolding of a $\phi_{0} \in I(k)$ of $L$-corank $k$.

Similarly we have the following, which is needed in $\S 3$.

Proposition 1.3. Let $\phi_{\lambda}: X^{n}, x_{\lambda} \rightarrow M^{2 n}, \phi_{\lambda}\left(x_{\lambda}\right)\left(\operatorname{resp} . T^{*}\left(Q^{n}\right), \phi_{\lambda}\left(x_{\lambda}\right)\right)$ be an $\ell-$ parameter $C^{\infty}$ family of isotropic map-germs. Assume the corank of $\phi_{0}$ (res. the L-corank of $\phi_{0}$, i.e. the corank of $\left.\pi \circ \phi_{0}\right)$ is equal to $k$. Then $\phi_{\lambda}$ is symplectically (resp. Lagrange) equivalent by an $\ell$-parameter family of symplectic (resp. Lagrange) equivalences to an 
$\ell$-parameter family of isotropic unfoldings of an $\ell$-parameter family $\phi_{\lambda}^{\prime}$ of elements in $I(k)$ with corank $\phi_{0}^{\prime}=k\left(\right.$ resp. L-corank $\left.\phi_{0}^{\prime}=k\right)$.

By Proposition 1.1 (resp. 1.2), in particular, an isotropic map-germ of corank one (resp. of L-corank one) is symplectically (resp. Lagrange) equivalent to an isotropic unfolding of a map-germ $\phi: \mathbf{R}, 0 \rightarrow T^{*} \mathbf{R}, 0$. Remark that $\phi$ is automatically isotropic, since any 2 -forms vanish on $\mathbf{R}, 0$, and, by the same reason, any deformation $\left(\phi_{s}, s\right)$ : $\mathbf{R} \times \mathbf{R}^{n-1}, 0 \rightarrow T^{*} \mathbf{R} \times \mathbf{R}^{n-1}, 0$ is also isotropic. Simply write $\phi_{s}(t)=(P(t, s), Q(t, s))$, $t \in \mathbf{R}, 0$. Then the corresponding isotropic unfolding is given by $\tilde{\Phi}=\left(\phi_{s} ; r, s\right)$, where

$$
r_{j}=\frac{\partial}{\partial s_{j}}\left(\int_{0}^{t} P \frac{\partial Q}{\partial t} d t\right)-P \frac{\partial Q}{\partial s_{j}}=\int_{0}^{t}\left(\frac{\partial P}{\partial s_{j}} \frac{\partial Q}{\partial t}-\frac{\partial P}{\partial t} \frac{\partial Q}{\partial s_{j}}\right) d t, \quad 1 \leq j \leq n-1 .
$$

Thus the description of isotropic map-germs of corank one (resp. of L-corank one) is easily handled: In fact the local symplectic classification of generic isotropic mappings of corank one is given in [I1], [Z]. (See also [G2].) Further the local Lagrange classification of generic isotropic mappings of L-corank one will be given in [I3].

To describe the structure of isotropic map-germs of corank one under Lagrange equivalence, we introduce the following notation:

Let $I, J$ be a decomposition of $\{1, \ldots, n-1\}: I \cup J=\{1, \ldots, n-1\}, \# I+\# J=n-1$. We fix a system of coordinates $\left(p_{I}, q_{J}, t\right)$ of $\mathbf{R}^{n}, 0$, where where $p_{I}=\left(p_{i}\right)_{i \in I}$ and so on. Then, for a pair of functions $u, v \in E_{n}$, we define a map-germ $\phi_{I, J}[u, v]: \mathbf{R}^{n}, 0 \rightarrow$ $T^{*} \mathbf{R}^{n}, 0$, by

$$
\begin{aligned}
p_{I} \circ \phi_{I, J}[u, v] & =p_{I}, \\
p_{J} \circ \phi_{I, J}[u, v] & =\int_{0}^{t}\left(\frac{\partial u}{\partial q_{J}} \frac{\partial v}{\partial t}-\frac{\partial u}{\partial t} \frac{\partial v}{\partial q_{J}}\right) d t, \quad p_{n} \circ \phi_{I, J}[u, v]=u . \\
q_{I} \circ \phi_{I, J}[u, v] & =\int_{0}^{t}\left(\frac{\partial u}{\partial t} \frac{\partial v}{\partial p_{I}}-\frac{\partial u}{\partial p_{I}} \frac{\partial v}{\partial t}\right) d t, \\
q_{J} \circ \phi_{I, J}[u, v] & =q_{J}, \quad q_{n} \circ \phi_{I, J}[u, v]=v .
\end{aligned}
$$

Then $\phi_{I, J}[u, v]$ is isotropic, of corank one and of L-corank \#I.

We need in $\S 3$ the following, which generalize Propositions 1.1, 1.2 and 1.3.

Proposition 1.4. Let $\phi_{\lambda}\left(\lambda \in \mathbf{R}^{\ell}, 0\right)$ be an $\ell$-parameter family of elements in $I(n)$ with corank $\phi_{0} \leq 1$. Then the family $\phi_{\lambda}$ is Lagrange equivalent to $\phi_{I, J}\left[u_{\lambda}, v_{\lambda}\right]$, for some decomposition $I, J$ of $\{1, \ldots, n-1\}$ and for some $u_{\lambda}, v_{\lambda}$ with $u_{\lambda}(0)=v_{\lambda}(0)=0$ (by a one-parameter family of Lagrange equivalences $\left.\left(\sigma_{\lambda}, \tau_{\lambda}\right)\right)$.

Moreover, if $\phi_{0}=\tau \circ \phi_{I, J}[u, v] \circ \sigma^{-1}$, then we can choose $\left(u_{\lambda}, v_{\lambda}, \sigma_{\lambda}, \tau_{\lambda}\right)$ so that $\left(u_{0}, v_{0}, \sigma_{0}, \tau_{0}\right)=(u, v, \sigma, \tau)$.

Proof. Notice that, for the case of Lagrange immersions, a similar result is wellknown (cf. $[\mathrm{AGV}]$ ).

First we see $\phi_{\lambda}$ is Lagrange equivalent to $\phi_{\lambda}^{\prime}$ of the following form:

$$
\phi_{\lambda}^{\prime}:\left(p_{I}, q_{J}, t\right) \mapsto\left(p_{I}, p_{J}, p_{n} ; q_{I}, q_{J}, q_{n}\right),
$$


for some fixed decomposition $I, J$ of $\{1, \ldots, n-1\}$, where $p_{J}, p_{n}, q_{I}, q_{n}$ are function-germs of $\left(p_{I}, q_{J}, t ; \lambda\right)$ with $\left.p_{J}\right|_{t=0}=\left.p_{n}\right|_{t=0}=\left.q_{I}\right|_{t=0}=\left.p_{n}\right|_{t=0}=0$.

Then there exists function-germ $e^{\prime}$ of $\left(p_{I}, q_{J}, t ; \lambda\right)$ with $\left.e^{\prime}\right|_{t=0}=0$ and

$$
d_{\left(p_{I}, q_{J}, t\right)} e^{\prime}=p_{I} d q_{I}+p_{J} d q_{J}+p_{n} d q_{n}=d\left(p_{I} q_{I}\right)-q_{I} d p_{I}+p_{J} d q_{J}+p_{n} d q_{n} .
$$

Set $e=e^{\prime}-p_{I} q_{I}$. Then $d_{\left(p_{I}, q_{J}, t\right)} e=-q_{I} d p_{I}+p_{J} d q_{J}+p_{n} d q_{n}$. Thus we have

$$
\frac{\partial e}{\partial p_{I}}=-q_{I}+p_{n} \frac{\partial q_{n}}{\partial p_{I}}, \quad \frac{\partial e}{\partial q_{J}}=p_{J}+p_{n} \frac{\partial q_{n}}{\partial q_{J}}, \quad \frac{\partial e}{\partial t}=p_{n} \frac{\partial q_{n}}{\partial t},
$$

and we see $e=\int_{0}^{t} p_{n}\left(\partial q_{n} / \partial t\right) d t$. Hence

$$
\begin{aligned}
& q_{I}=-\frac{\partial e}{\partial p_{I}}+p_{n} \frac{\partial q_{n}}{\partial p_{I}}=\int_{0}^{t}\left(\frac{\partial p_{n}}{\partial t} \frac{\partial q_{n}}{\partial p_{I}}-\frac{\partial p_{n}}{\partial p_{I}} \frac{\partial q_{n}}{\partial t}\right) d t, \\
& p_{J}=\frac{\partial e}{\partial q_{J}}-p_{n} \frac{\partial q_{n}}{\partial q_{J}}=\int_{0}^{t}\left(\frac{\partial p_{n}}{\partial q_{J}} \frac{\partial q_{n}}{\partial t}-\frac{\partial p_{n}}{\partial t} \frac{\partial q_{n}}{\partial q_{J}}\right) d t .
\end{aligned}
$$

If we set $u_{\lambda}=p_{n}(\cdot, \lambda)$ and $v_{\lambda}=q_{n}(\cdot, \lambda)$, then we have the required result.

2. Isotropic jets. Recall that $I(n)$ denotes the set of isotropic map-germs $\mathbf{R}^{n}, 0 \rightarrow$ $T^{*} \mathbf{R}^{n}, 0$. Set $J^{k}(n)=\left\{j^{k} \phi(0) \mid \phi: \mathbf{R}^{n}, 0 \rightarrow T^{*} \mathbf{R}^{n}, 0\right\}=J^{k}(n, 2 n)$. A $k$-jet $z \in J^{k}(n)$ is called isotropic if $z=j^{k} \phi(0)$ for some $\phi \in I(n)$. We denote by $J_{I}^{k}(n)$ the set of isotropic $k$-jets in $J^{k}(n)$, for $k=1,2, \ldots, \infty$.

Our problem in this section is to study the structure of the set $J_{I}^{k}(n)$ of isotropic $k$-jets.

We denote by $S^{k}(n)$ (resp. $\left.L^{k}(n)\right)$ the group of pairs $\left(j^{k} \sigma(0), j^{k} \tau(0)\right)$ of $k$-jets of a diffeomorphism-germ $\sigma: \mathbf{R}^{n}, 0 \rightarrow \mathbf{R}^{n}, 0$ and a symplectic (resp. Lagrange, i.e. fiberpreserving symplectic) diffeomorphism-germ $\tau: T^{*} \mathbf{R}^{n}, 0 \rightarrow T^{*} \mathbf{R}^{n}, 0$. Naturally $S^{k}(n)$, therefore $L^{k}(n)$ acts on $J^{k}(n)$. Then $J_{I}^{k}(n)$ is a $S^{k}(n)$-invariant, therefore $L^{k}(n)$-invariant subset of $J^{k}(n)$.

We introduce an auxiliary notion:

Definition 2.1. A map-germ $\phi: \mathbf{R}^{n}, 0 \rightarrow T^{*} \mathbf{R}^{n}, 0$ is called $\ell$-isotropic $(\ell=1,2, \ldots$ $\ldots, \infty)$ if $\phi^{*} \omega \in m_{n}^{\ell} \Omega$, that is, $j^{\ell-1}\left(\phi^{*} \omega\right)(0)=0$, where $\Omega$ denotes the $E_{n}$-module of germs of differential 2-form on $\mathbf{R}^{n}, 0$. A jet $z \in J^{k}(n)$ is called $\ell$-isotropic if $z=j^{k} \phi(0)$ for some $\ell$-isotropic $\phi$.

Now set $J_{\ell-I}^{k}(n)=\left\{z \in J^{r}(n) \mid z\right.$ is $\ell$-isotropic $\}$. Then we have a sequence of sets:

$$
J^{k}(n) \supset J_{1-I}^{k}(n) \supset \ldots \supset J_{k-I}^{k}(n) \supset J_{k+1-I}^{k}(n) \supset \ldots \supset J_{\infty-I}^{k}(n) \supset J_{I}^{r}(n) .
$$

Then it is easy to see the following lemmata:

Lemma 2.2. $J_{\ell-I}^{k}(n)$ is algebraic (resp. semi-algebraic) if $\ell \leq k($ resp. $k<\ell<\infty)$.

LEMma 2.3. $J_{1-I}^{1}(n)=J_{I}^{1}(n)$, which is identified with the set of linear isotropic mappings $\mathbf{R}^{n} \rightarrow T^{*} \mathbf{R}^{n}$. Moreover $J_{I}^{1}(n) \subset$ Hom $_{\mathbf{R}}\left(\mathbf{R}^{n}, \mathbf{R}^{2 n}\right) \cong \mathbf{R}^{2 n^{2}}$ is a quadratic hypersurface with Sing $J_{I}^{1}(n)=\{$ linear isotropic mappings of corank $\geq 2\}$. ([G1], [A2]. See also Example 2.4 below.) 
Set $\Sigma^{i}(n)=\left\{j^{1} \phi(0) \in J^{1}(n) \mid\right.$ corank $\left.\phi=i\right\}$ and $\Sigma_{i}(n)=\left\{j^{1} \phi(0) \in J^{1}(n) \mid\right.$ L-corank $\phi=i\}$.

The natural projection $\pi_{k, \ell}: J^{k}(n) \rightarrow J^{\ell}(n)(k \geq \ell)$ induces $\pi_{k, \ell}: J_{I}^{k}(n) \rightarrow J_{I}^{\ell}(n)$. Denote the inverse image $\pi_{k, 1}^{-1}\left(\Sigma^{i}(n)\right)$ (resp. $\left.\pi_{k, 1}^{-1}\left(\Sigma_{i}(n)\right)\right) \subset J^{k}(n)$ also by $\Sigma^{i}(n)$ (resp. $\left.\Sigma_{i}(n)\right)$.

Now, to study (first order) singularities or Lagrange singularities of isotropic mappings, set $\Sigma_{I}^{i}(n)=\Sigma^{i}(n) \cap J_{I}^{1}(n)\left(\right.$ resp. $\left.\Sigma_{j, I}(n)=\Sigma_{j}(n) \cap J_{I}^{1}(n)\right)$. Further set $\Sigma_{j, I}^{i}(n)=$ $\Sigma_{I}^{i}(n) \cap \Sigma_{j, I}(n)(i \leq j)$. We freely regard $\Sigma_{I}^{i}(n), \Sigma_{j, I}(n)$ and $\Sigma_{j, I}^{i}(n)$ as subsets in $J_{I}^{k}(n)$ for arbitrary $k$.

Remark that $\Sigma_{I}^{0}(n)$ is the set of jets of Lagrangian immersions $\mathbf{R}^{n}, 0 \rightarrow T^{*} \mathbf{R}^{n}, 0$.

EXAmple 2.4. To describe $J_{I}^{1}(n)$, we set $V=\operatorname{Hom}_{\mathbf{R}}\left(\mathbf{R}^{n}, \mathbf{C}^{n}\right) \cong M_{n}(\mathbf{C})$, the space of complex square matrices of size $n$. We denote by $\langle\cdot, \cdot\rangle$ the standard Hermitian structure on $\mathbf{C}^{n}$, and by $[\cdot, \cdot]$ the standard symplectic structure on $\mathbf{C}^{n} ;[u, v]=\operatorname{Im}\langle u, v\rangle, u, v \in \mathbf{C}^{n}$. Then $J_{I}^{1}(n)$ is identified with the set $W \subset V$ of linear isotropic mappings. We define a polynomial map $\rho: V \rightarrow \operatorname{Alt}(n)$ by $\rho(\ell)(u, v)=[\ell u, \ell v], u, v \in \mathbf{R}^{n}$. where $\operatorname{Alt}(n)$ is the space of skewsymmetric bilinear forms $\mathbf{R}^{n} \times \mathbf{R}^{n} \rightarrow \mathbf{R}$. Then we see $W=\rho^{-1}(O)$ and therefore $W$ is a real algebraic subset of $V$. Further we see that $\rho$ is submersive along $\Sigma_{I}^{0}(n) \cup \Sigma_{I}^{1}(n)$. Hence $\operatorname{Sing}(W)$ is contained in $\bigcup_{j=2}^{n} \Sigma_{I}^{j}(n)$. Furthermore each $\Sigma_{I}^{i}(n)$ is a submanifold of $V$ of dimension $(1 / 2)\{n(3 n+1)-i(3 i+1)\}, 0 \leq i \leq n$. (See [I2]).

Now we pick up a wider domain than $\Sigma_{I}^{0}(n)$ in $J_{I}^{k}(n)$ :

$$
R^{k}(n)=\left\{j^{k} \phi(0) \in J_{I}^{k}(n) \mid \operatorname{corank} \phi \leq 1\right\}=\Sigma_{I}^{0}(n) \cup \Sigma_{I}^{1}(n),
$$

which is open in $J_{I}^{k}(n)$.

EXAMPLE 2.5. Let $n=2$. The ambient space $J^{1}(2)=J^{1}(2,4)$ is identified with $\mathbf{R}^{8}$, by setting $q_{1}=a x+b y, q_{2}=c x+d y ; p_{1}=\alpha x+\beta y, p_{2}=\gamma x+\delta y$, where $(x, y)$ is the coordinate of $\mathbf{R}^{2}$. Then $J_{I}^{1}(2)$ is given by $\alpha b-\beta a+\gamma d-\delta c=0$. Thus $\operatorname{dim} J_{I}^{1}(2)=7$ and $\operatorname{Sing} J_{I}^{1}(2)=$ $\{0\}$. Set $\bar{\Sigma}_{I}^{1}(2)=\Sigma_{I}^{1}(2) \cup \Sigma_{I}^{2}(2)$. This is defined by $\alpha b-\beta a+\gamma d-\delta c=a d-b c=\alpha \delta-\beta \gamma=0$, which is of dimension 5 and $\operatorname{Sing} \bar{\Sigma}_{I}^{1}(2)=\{0\}$. Further set $\bar{\Sigma}_{1, I}(2)=\Sigma_{1, I}(2) \cup \Sigma_{2, I}(2)$. Then $\bar{\Sigma}_{1, I}(2)$ is defined by $\alpha b-\beta a+\gamma d-\delta c=a d-b c=0$, which is of dimension 6 and $\operatorname{Sing} \bar{\Sigma}_{1, I}(2)=\{a=b=c=d=0\}$. Now set $X_{0}=J_{I}^{1}(2), X_{1}=J^{1}(2) \cap\{a d-b c=$ $0\}=\bar{\Sigma}_{1, I}(2), X_{2}=X_{1} \cap\{\alpha \delta-\beta \gamma=0\}=\bar{\Sigma}_{I}^{1}(2), X_{3}=\{a=b=c=d=0\}=\Sigma_{2, I}(2)$, $X_{4}=X_{2} \cap X_{3}=\Sigma_{2, I}(2) \cap \Sigma_{I}^{1}(2)=\Sigma_{2, I}^{1}(2) \cup\{O\}$, and $X_{7}=\{O\}=\Sigma_{I}^{2}(2)$. Further set $U_{0}=X_{0}-X_{1}, U_{1}=X_{1}-\left(X_{2} \cup X_{3}\right), U_{2}=X_{2}-X_{4}, U_{3}=X_{3}-X_{4}, U_{4}=X_{4}-X_{7}=\Sigma_{2, I}^{1}(2)$. Then $\left\{U_{0}, U_{1}, U_{2}, U_{3}, U_{4}\right\}$ is the stratification of $R^{1}(2)$ by the orbits of $L^{1}(2)$, and we see $\operatorname{codim} U_{j}=j$.

The following is the key to study the structure of $R^{k}(n)$ :

Proposition 2.6. $J_{k-I}^{k}(n) \cap\left(\Sigma^{0}(n) \cup \Sigma^{1}(n)\right)=R^{k}(n)$.

Pr o of. Consider the natural action of $S^{k}(n)$ on $J^{k}(n)$ preserving $J_{I}^{k}(n)$ and $J_{\ell-I}^{k}(n)$. Assume $z$ is a $k$-isotropic $k$-jet and corank $z \leq 1$. Then, by Proposition 1.1, there exist polynomials $P_{1}, \ldots, P_{n}$ and $Q_{n}$ of degree $\leq k$ such that $z$ is (symplectically) equivalent to $j^{k}\left(P ; x^{\prime}, Q_{n}\right)(0)$, where $P=\left(P_{1}, \ldots, P_{n}\right)$ and $x^{\prime}=\left(x_{1}, \ldots, x_{n-1}\right)$. The map-germ 
$\phi=\left(P ; x^{\prime}, Q_{n}\right)$ is also $k$-isotropic, therefore, $\phi^{*} \omega=\sum_{i=1}^{n-1} d P_{i} d x_{i}+d P_{n} d Q_{n} \in m_{n}^{k} \Omega$. Comparing coefficients of $d x_{i} \wedge d x_{n}$, we have

$$
\frac{\partial P_{i}}{\partial x_{n}}=\frac{\partial\left(P_{n}, Q_{n}\right)}{\partial\left(x_{i}, x_{n}\right)}+\rho_{i}, \quad \rho_{i} \in m_{n}^{k} \quad(1 \leq i \leq n-1) .
$$

Set $\tilde{P}_{i}=P_{i}-\int_{0}^{x_{n}} \rho_{i} d x_{n}, 1 \leq i \leq n-1, \tilde{P}=\left(\tilde{P}_{1}, \ldots, \tilde{P}_{n-1}, P_{n}\right)$ and $\phi^{\prime}=\left(\tilde{P} ; x^{\prime}, Q_{n}\right)$. Then $j^{k} \phi^{\prime}(0)=z$ and $\phi^{\prime}$ is isotropic. Hence $z$ is isotropic; $z \in J_{I}^{k}(n)$. So $z \in J_{I}^{k}(n) \cap$ $\left(\Sigma^{0}(n) \cup \Sigma^{1}(n)\right)=R^{k}(n)$.

The converse inclusion is clear.

Modifying the proof of Lemma 2.6, we have

Lemma 2.7. Let $a: \mathbf{R}^{\ell}, 0 \rightarrow J^{k}(n)$ be a $C^{\infty}$-map-germ with $a\left(\mathbf{R}^{\ell}, 0\right) \subset R^{k}(n)$. Let $a(0)=j^{k} \phi(0)$ for some $\phi \in I(n)$. Then there exists a $C^{\infty}$ family $\phi_{\lambda} \in I(n), \lambda \in \mathbf{R}^{\ell}, 0$, such that $j^{k} \phi_{\lambda}(0)=a(\lambda)$ and $\phi_{0}=\phi$.

In particular, for a $C^{\infty}$-curve $z_{\lambda}$ in $R^{k}(n)$ through $z_{0}=j^{k} \phi(0), \phi \in I(n)(\lambda \in \mathbf{R}, 0)$, there exists a $C^{\infty}$ family $\phi_{\lambda}$ of $I(n)$ with $j^{k} \phi_{\lambda}(0)=z_{\lambda}$ and $\phi_{0}=\phi$.

Pro of. By Proposition 1.1, using a symplectic equivalence, we may assume $\phi$ is of type $\left(P ; x^{\prime}, Q_{n}\right)$, where $P=\left(P_{1}, \ldots, P_{n}\right)$ and $P_{1}, \ldots, P_{n}, Q_{n} \in m_{n}$. Then there exist a (not necessarily isotropic) deformation $\left(P_{\lambda}, x^{\prime}, Q_{n \lambda}\right)$ of $\phi$ such that $j^{k}\left(P_{\lambda}, x^{\prime}, Q_{n \lambda}\right)(0)$ is symplectically equivalent to $a(\lambda)$ by a family of symplectic equivalences $\left(\sigma_{\lambda}, \tau_{\lambda}\right)$ with $\left(\sigma_{0}, \tau_{0}\right)$. By the same procedure as in the proof of Proposition 2.6, we find an isotropic deformation $\phi_{\lambda}^{\prime}=\left(\tilde{P}_{\lambda}, x^{\prime}, \tilde{Q}_{n \lambda}\right)$ of $\phi$ such that $j^{k} \phi_{\lambda}^{\prime}(0)=j^{k}\left(P_{\lambda}, x^{\prime}, Q_{n \lambda}\right)(0)$. Applying the inverse of $\left(\sigma_{\lambda}, \tau_{\lambda}\right)$ to $\phi_{\lambda}^{\prime}$, we get the required family $\phi_{\lambda}$.

Remark 2.8. In general it seems natural to pose the following conjecture: For any $k<\infty$, there exists $\ell=\ell(k)<\infty$, such that $J_{\ell-I}^{k}(n)=J_{I}^{k}(n)$. Further, $J_{\infty-I}^{\infty}(n)=$ $J_{I}^{\infty}(n)$.

3. Transversality Theorem. We fix $n \geq 1$. In this section, we denote by $V^{k}$ the affine space $J^{k}(n)=J^{k}(n, 2 n)$ of $k$-jets of $C^{\infty}$ map-germs from $\mathbf{R}^{n}, 0$ to $T^{*} \mathbf{R}^{n}, 0$. Besides, we denote by $\Lambda^{k}$ the vector space of $(k-1)$-jets of closed 2 -forms in $\mathbf{R}^{n}, 0$. Remark that, by Poincaré lemma, $\Lambda^{k}$ is a vector subspace of the vector space of $(k-1)$-jets of 2 -forms in $\mathbf{R}^{n}, 0$ and it is equal to the space of $(k-1)$-jets of exact 2 -forms in $\mathbf{R}^{n}, 0$. Furthermore we see

$$
\operatorname{dim} \Lambda^{k}={ }_{n+k} C_{k} n-{ }_{n+k+1} C_{k+1}+1 .
$$

We endow $T^{*} \mathbf{R}^{n}=\mathbf{R}^{2 n}$ with the standard symplectic form $\omega=\sum_{i=1}^{n} d p_{i} \wedge d q_{i}$. Then define the polynomial mapping $\rho: V^{k} \rightarrow \Lambda^{k}$ by $\rho\left(j^{k} \phi(0)\right)=j^{k-1}\left(\phi^{*} \omega\right)(0)$.

First we show

Lemma 3.1. For a $z \in V^{k}$, consider the tangent mapping $\rho_{*}: T_{z} V^{k}\left(\cong V^{k}\right) \rightarrow$ $T_{\rho(z)} \Lambda^{k}\left(\cong \Lambda^{k}\right)$. If corank $(z) \leq 1$, then $\rho_{*}$ is surjective, that is, $\rho$ is a submersion at $z$.

Proof. Set $z=j^{k} \phi(0)$ and $\phi=(P, Q)=\left(P_{1}, \ldots, P_{n} ; Q_{1}, \ldots, Q_{n}\right)$. For a oneparameter deformation $\phi_{t}=(P+t \tilde{P}, Q+t \tilde{Q})$, we have

$$
d\left(\phi^{*} \omega\right) /\left.d t\right|_{t=0}=\sum_{i} d \tilde{P}_{i} d Q_{i}-\sum_{i} d \tilde{Q}_{i} d P_{i}=d\left(\sum_{i}\left(\tilde{P}_{i} d Q_{i}-\tilde{Q}_{i} d P_{i}\right)\right) .
$$


Therefore $\rho_{*}(\tilde{P}, \tilde{Q})=j^{k-1}\left\{d\left(\sum_{i}\left(\tilde{P}_{i} d Q_{i}-\tilde{Q}_{i} d P_{i}\right)\right)\right\}(0)$. Then, to show $\rho_{*}$ is surjective, it is sufficient to show that, for any one-form germ $E$ on $\mathbf{R}^{n}, 0$, there exist function-germs $\tilde{P}_{i}, \tilde{Q}_{i}, 1 \leq i \leq n$, and $e$ such that

$$
\sum_{i}\left(\tilde{P}_{i} d Q_{i}-\tilde{Q}_{i} d P_{i}\right)=E+d e
$$

Since the property that $\rho_{*}$ is surjective is $S^{k}(n)$-invariant, we may assume

$$
Q=\left(x_{1}, \ldots, x_{n-1}, u\left(x^{\prime}, x_{n}\right)\right),
$$

where $x^{\prime}=\left(x_{1}, \ldots, x_{n-1}\right)$. Set $E=\sum_{i=1}^{n} E_{i} d x_{i}$. If we set, in particular, $\tilde{Q}=0$ and $\tilde{P}_{n}=0$, then the equation to solve turns out to

$$
\sum_{i=1}^{n-1}\left(\tilde{P}_{i}-E_{i}-\partial e / \partial x_{i}\right) d x_{i}-\left(E_{n}+\partial e / \partial x_{n}\right) d x_{n}=0 .
$$

First set $e=-\int_{0}^{x_{n}} E_{n} d x_{n}$. Then next set $\tilde{P}_{i}=E_{i}+\partial e / \partial x_{i}, i=1, \ldots, n-1$. Thus $\rho_{*}$ is surjective if $\operatorname{corank}(z) \leq 1$.

Proposition 3.2. $R^{k}(n)$ is a $S^{k}(n)$-invariant submanifold of $V^{k}=J^{k}(n, 2 n)$. The codimension of $R^{k}(n)$ in $V^{k}$ is equal to $\operatorname{dim} \Lambda^{k}$. Moreover the natural projection $\pi_{k, k-1}$ : $R^{k}(n) \rightarrow R^{k-1}(n)$ is a surjective submersion $(k \geq 1)$.

Corollary 3.3. For an n-manifold $X$ and a symplectic $2 n$-manifold $M$, the set $R^{k}(X, M)$ of $k$-jets $j^{k} \phi(x)$ of isotropic map-germs $\phi: X, x \rightarrow M$ of corank $\leq 1$ is a submanifold of $J^{k}(X, M)$ of codimension $\operatorname{dim} \Lambda^{k}$. Moreover, the natural projection $\pi_{k, k-1}: R^{k}(X, M) \rightarrow R^{k-1}(X, M)$ is a surjective submersion $(k \geq 1)$.

Remark 3.4. The system $\left\{R^{k}\right\}$ is regarded as the prolongation of the non-linear first order partial differential equation: $\omega=0$ ([Go]).

Proof of Proposition 3.2. The first part is clear from Lemma 3.1 and Proposition 2.6.

To see the second part, take $z \in R^{k}(n)$ and a $C^{\infty}$ curve in $R^{k-1}(n)$ through $\pi_{k, k-1} z \in$ $R^{k-1}(n)$. Let $j^{k} \phi(0)=z$ for a $\phi \in I(n)$. Then, by Lemma 2.7, this curve lifts to a $C^{\infty}$ deformation $\phi_{\lambda}$ of $\phi$ in $I(n)$. Taking $k$-jets of $\phi_{\lambda}$, we have a $C^{\infty}$ lifting of the curve through $z$.

Proof of Theorem 0.1. We follow the standard argument to prove the transversality theorem, for instance, as in $\S 3$ of [M1].

First of all we remark the following: The space $C_{I}^{\infty}(X, M)$ of isotropic mappings is a Baire space with respect to the Whitney $C^{\infty}$ topology. Furthermore $C_{I}^{\infty}(X, M)^{1} \subset$ $C_{I}^{\infty}(X, M)$ is also a Baire space.

The proof of this fact is similar to that of Proposition 3.1 of [M1]: The point is that the isotropic condition for a mapping is a local and closed condition about its one-jet section. The second half is clear, because $C_{I}^{\infty}(X, M)^{1}$ is open in $C_{I}^{\infty}(X, M)$.

Then to show Theorem 0.1 , it is sufficient to prove that, for each $\phi \in C_{I}^{\infty}(X, M)^{1}$ and for each $x_{0} \in X$, there exist a manifold $E, e_{0} \in E$, and a continuous mapping $\varphi:\left(E, e_{0}\right) \rightarrow\left(C_{I}^{\infty}(X, M)^{1}, \phi\right)$ such that the induced mapping $\Phi: E \times X \rightarrow R^{k}(X, M)$ 
defined by $\Phi(e, x)=j^{k}(\varphi(e))(x)$ is submersive at $\left(e_{0}, x_{0}\right)$. (See Lemma 3.2 and the argument in Proposition 3.3 of [M1].)

To complete this, we use the results in $\S \S 1$ and 2 as follows: Denote by $L(n)$ the group of Lagrange equivalences on $I(n)$. For each decomposition $I, J$ of indices $\{1, \ldots, n-1\}$, we define a map $\Psi: m_{n} \times m_{n} \times L(n) \rightarrow I(n)$ by $\Psi(u, v ; \sigma, \tau)=\tau \circ \phi_{I, J}[u, v] \circ \sigma^{-1}$. The mapping $\Psi$ induces a $C^{\infty}$ mapping $\Psi^{k}: J^{k}(n, 2) \times L^{k}(n) \rightarrow R^{k}(n)$ by $\Psi^{k}\left(j^{k} u(0), j^{k} v(0)\right.$; $\left.j^{k} \sigma(0), j^{k} \tau(0)\right)=j^{k}(\Psi(u, v ; \sigma, \tau))(0)$.

Then $\Psi^{k}$ is a submersion: In fact, let $(u, v, \sigma, \tau) \in m_{n} \times m_{n} \times L(n), \Psi(u, v, \sigma, \tau)=\phi \in$ $I(n)$ and $z=j^{k} \phi(0) \in R^{k}(n)$. Let $z_{\lambda}$ be a smooth curve in $R^{k}(n)$ through $z$. Then, by Lemma 2.7, there exists a $C^{\infty}$-deformation $\phi_{\lambda}$ in $I(n)$ of $\phi$ with $j^{k} \phi_{\lambda}(0)=z_{\lambda}$. Then by Proposition 1.4, there exist $u_{\lambda}, v_{\lambda}, \sigma_{\lambda}, \tau_{\lambda}$ such that $\Psi\left(u_{\lambda}, v_{\lambda} ; \sigma_{\lambda}, \tau_{\lambda}\right)\left(=\tau_{\lambda} \circ \phi_{I, J}\left[u_{\lambda}, v_{\lambda}\right] \circ\right.$ $\left.\sigma_{\lambda}^{-1}\right)=\phi_{\lambda}$ and that $\left(u_{0}, v_{0}, \sigma_{0}, \tau_{0}\right)=(u, v, \sigma, \tau)$. So we have

$$
\Psi^{k}\left(j^{k} u_{\lambda}(0), j^{k} v_{\lambda}(0) ; j^{k} \sigma_{\lambda}(0), j^{k} \tau_{\lambda}(0)\right)=z_{\lambda}
$$

This means that any $C^{\infty}$ curve on $R^{k}(n)$ through $z$ has a $C^{\infty}$ lifting with respect to $\Psi^{k}$, and therefore $\Psi^{k}$ is a submersion.

We identify $J^{k}(n, 2)$ with the space pf pairs of polynomials of $n$ variables with degree $\leq k$ without constant terms. We set then $E=J^{k}(n, 2) \times L^{k}(n) \times M$.

Let $\phi \in C_{I}^{\infty}(X, M)^{1}$ and $x_{0} \in X$. Then we have

$$
\phi_{x_{0}}=\tau_{0} \circ \phi_{I, J}\left[u_{0}, v_{0}\right] \circ \sigma_{0}^{-1}: X, x_{0} \rightarrow M, \phi\left(x_{0}\right),
$$

for a diffeomorphism-germ $\sigma_{0}: \mathbf{R}^{n}, 0 \rightarrow X, x_{0}$, for a symplectic diffeomorphism-germ $\tau_{0}: T^{*} \mathbf{R}^{n}, 0 \rightarrow M, \phi\left(x_{0}\right)$, for a decomposition $I, J$ of $\{1,2, \ldots, n-1\}$, and for a pair of elements $u_{0}, v_{0} \in E_{n}$. (See Proposition 1.4.) Set $e_{0}=\left(u_{0}, v_{0}, 1, \phi\left(x_{0}\right)\right)$, where $1 \in L^{k}(n)$ is the unit, and construct $\varphi:\left(E, e_{0}\right) \rightarrow\left(C_{I}^{\infty}(X, M)^{1}, \phi\right)$ by

$$
\varphi(u, v ; \sigma, \tau ; m)=\tau_{0} \circ\left\{b \cdot T_{m} \circ \tau \circ \phi_{I, J}[u, v] \circ \sigma^{-1}+(1-b) \tau_{0}^{-1} \circ \phi \sigma_{0}\right\} \circ \sigma_{0}^{-1},
$$

where $b$ is a bump function on $J^{k}(n, 2) \times L^{k}(n) \times \mathbf{R}^{2 n} \times \mathbf{R}^{n}$, which is equal to 1 near the origin and is equal to 0 off a neighbourhood of the origin, and $T_{m}: T^{*} \mathbf{R}^{n} \rightarrow T^{*} \mathbf{R}^{n}$ is the translation by $\tau_{0}^{-1}(m)-\tau_{0}^{-1}\left(\phi\left(x_{0}\right)\right)$.

Then $\phi$ is clearly continuous and we have, near $x_{0}$,

$$
\varphi(u, v ; \sigma, \tau ; m)=\tau_{0} \circ T_{m} \circ \tau \circ \phi_{I, J}[u, v] \circ \sigma^{-1} \circ \sigma_{0}^{-1} .
$$

Since, as verified above, $\Psi^{k}$ is a submersion, $\Phi$ constructed from $\varphi$ is a submersion at $\left(e_{0}, x_{0}\right)$.

Re m a r k 3.5. By the same method as in the proof of Theorem 0.1, we can show also the multi-transversality theorem for isotropic mappings of corank at most one (cf. [M1]).

Lastly, we give a simple consequence of Theorem:

Corollary 3.6. Let $n<4$. Then generic isotropic map-germs $\phi: \mathbf{R}^{n}, 0 \rightarrow T^{*} \mathbf{R}^{n}, 0$ of corank one are of $L$-corank one.

For the proof of Corollary 3.6, we repeat the constructions in $\S 1$, in the first jet level.

Denote by $J^{1}\left(n-k, R^{1}(k)\right)$ the space of 1-jets of map-germs $\mathbf{R}^{n-k}, 0 \rightarrow R^{1}(k) \subset J^{1}(k)$. Then define a mapping $\delta: J^{1}\left(n-k, R^{1}(k)\right) \rightarrow R^{1}(n)$ as follows: Let $j^{1} \alpha(0) \in J^{1}(n-$ 
$\left.k, R^{1}(k)\right)$ with $\alpha: \mathbf{R}^{n-k}, 0 \rightarrow R^{1}(k)$. Denote by $A: \mathbf{R}^{n-k} \times \mathbf{R}^{k}, 0 \rightarrow T^{*} \mathbf{R}^{k}, 0$ the induced deformation. Then there exists $e: \mathbf{R}^{n-k} \times \mathbf{R}^{k}, 0 \rightarrow \mathbf{R}$ such that $d e_{s}=A_{s}^{*} \theta, s \in \mathbf{R}^{n-k}, 0$. If we assume $e(0, s)=0$, then $e$ is uniquely determined. From

$$
d e=\sum_{i} p_{i} \circ A_{s} d\left(q_{i} \circ A_{s}\right)+\sum_{i}\left(\partial e / \partial s_{i}\right) d s_{i}
$$

we define an isotropic map-germ $B: \mathbf{R}^{n-k} \times \mathbf{R}^{k}, 0 \rightarrow T^{*}\left(\mathbf{R}^{n-k} \times \mathbf{R}^{k}\right), 0$, by

$$
p_{i} \circ B=p_{i} \circ A, \quad q_{i} \circ B=q_{i} \circ A, \quad r_{i} \circ B=\partial e / \partial s_{i}, \quad s_{i} \circ B=s_{i} .
$$

Clearly $j^{1} B(0)$ depends only on the one-jet $j^{1} \alpha(0)$. Then set $\delta\left(j^{1} \alpha(0)\right)=j^{1} B(0)$.

From $\delta$, we define

$$
\Delta: J^{1}\left(n-k, R^{1}(k)\right) \times L^{1}(n) \rightarrow R^{1}(n) \times L^{1}(n) \rightarrow R^{1}(n),
$$

where the second mapping is the $L^{1}(n)$-action on $R^{1}(n)$. Then we easily verify the following

LEMMA 3.7. (1) $\Delta$ is a submersion onto $R^{1}(n)-\bar{\Sigma}_{k+1, I}(n)$.

(2) $\Delta^{-1}\left(\Sigma_{j, I}(n)\right)=\operatorname{Pr}^{-1}\left(\Sigma_{j, I}(k)\right) \times L^{1}(n), 0 \leq j \leq k$.

(3) $\Delta^{-1}\left(\Sigma_{I}^{1}(n)\right)=\operatorname{Pr}^{-1}\left(\Sigma_{I}^{1}(k)\right) \times L^{1}(n)$.

(4) $\operatorname{codim} \Sigma_{j, I}(n)=\operatorname{codim} \Sigma_{j, I}(k)$ and $\operatorname{codim} \Sigma_{j, I}^{1}(n)=\operatorname{codim} \Sigma_{j, I}^{1}(k), 0 \leq j \leq k$.

Here $\operatorname{Pr}: J^{1}\left(n-k, R^{1}(k)\right) \rightarrow R^{1}(k)$ is the natural projection.

From Lemma 3.7 and Example 2.5, we have

LEMMA 3.8. $\operatorname{codim} \Sigma_{2, I}^{1}(n)=\operatorname{codim} \Sigma_{2, I}^{1}(2)=4$.

Proof of Corollary 3.6. By Theorem 0.1 (or Corollary 0.2) and Lemma 3.8, a generic isotropic mapping of corank at most one has no singularity of type $\Sigma_{2, I}^{1}(n)$ if $n<4$. Therefore a generic isotropic map-germ of corank one is necessarily of L-corank one, if $n<4$.

We conclude this paper by showing the following:

Proposition 3.9. There exists a generic isotropic map-germ $\phi: \mathbf{R}^{4}, 0 \rightarrow T^{*} \mathbf{R}^{4}$ such that corank $\phi=1$ and L-corank $\phi=2$.

Proof. It is sufficient to show that there exists an isotropic map-germ $\phi \in I(4)$ of corank one and of L-corank two such that the one-jet extension $j^{1} \phi: \mathbf{R}^{4}, 0 \rightarrow R^{1}(4)$ is transverse to $\Sigma_{2, I}^{1}(4)$ at 0 : By any isotropic perturbations of $\phi$, the Lagrange singular point of type $\Sigma_{2, I}^{1}$ does not vanish.

On the other hand, by Example 2.5 and Lemma 3.7, we easily have the following criterion for the transversality: Assume that $\phi \in I(4)$ is constructed from a two parameter isotropic deformation $\left(P_{1}, P_{2}, Q_{1}, Q_{2}\right)$ of an element of $I(2)$ as in $\S 1$, where $P_{1}, P_{2}, Q_{1}, Q_{2}$ are function-germs of $x, y, z, w$, and $z, w$ are regarded as parameters. If $\partial P_{1} / \partial x(0) \neq 0$, then $j^{1} \phi$ transversely intersects $\Sigma_{2, I}^{1}(4)$ at 0 , if and only if the map-germ

$$
f=\left(\frac{\partial P_{1}}{\partial x} \frac{\partial P_{2}}{\partial y}-\frac{\partial P_{2}}{\partial x} \frac{\partial P_{1}}{\partial y}, \frac{\partial Q_{1}}{\partial x}, \frac{\partial Q_{2}}{\partial x}, \frac{\partial Q_{2}}{\partial y}\right): \mathbf{R}^{4}, 0 \rightarrow \mathbf{R}^{4}, 0
$$

is of maximal rank. 
Then we define $\phi \in I(4)$, for instance, by

$$
\begin{gathered}
p_{1}=x, \quad p_{2}=(1 / 2) y^{2}, \quad p_{3}=-(1 / 2) x y^{2}, \quad p_{4}=-(1 / 3) y^{3}, \\
q_{1}=(1 / 2)\left(x^{2}+y^{2} z\right), \quad q_{2}=x z+y w+y^{2}, \quad q_{3}=z, \quad q_{4}=w .
\end{gathered}
$$

Remark that $e=(1 / 3) x^{3}+(1 / 4) y^{4}+(1 / 2) x y^{2} z+(1 / 6) y^{3} w$ is a generating function of $\phi$. In this example, $P_{1}=x, P_{2}=(1 / 2) y^{2}, Q_{1}=(1 / 2)\left(x^{2}+y^{2} z\right), Q_{2}=x z+y w+y^{2}$, and therefore $f=(y, x, z, w+2 y)$. Hence $\phi$ satisfies the required transversality condition.

\section{References}

[A1] V. I. Arnol'd, Lagrangian manifolds with singularities, asymptotic rays, and the open swallowtail, Functional Anal. Appl. 15 (4) (1981), 235-246.

[A2 - Singularities of Caustics and Wave Fronts, Kluwer Academic Publishers, 1990.

[AGV] V. I. Arnol'd, S. M. Gusein-Zade and A. N. Varchenko, Singularities of Differentiable Maps I, Birkhäuser, 1985.

[DP] N. H. Du'c et F. Pham, Germes de configurations Legendriennes stables et fonctions d'Airy-Weber généralisées, Ann. Inst. Fourier (Grenoble) 41 (4) (1991), 905-936.

[G1] A. B. Givental', Lagrangian imbeddings of surfaces and unfolded Whitney umbrella, Funktsional. Anal. i Prilozhen. 20 (3) (1986), 35-41 (in Russian).

[G2] - Singular Lagrangian varieties and their Lagrangian mappings, Itogi Nauki Tekh., Ser. Sovrem. Probl. Mat. 33, VINITI, 1988, 55-112 (in Russian).

[Go] H. Goldschmidt, Integrability criteria for systems of nonlinear partial differential equations, J. Differential Geom. 1 (1967), 269-307.

[I1] G. Ishikawa, The local model of an isotropic map-germ arising from one dimensional symplectic reduction, Math. Proc. Cambridge Philos. Soc. 111 (1992), 103-112.

[I2] - Maslov class of an isotropic map-germ arising from one-dimensional symplectic reduction, in: Adv. Stud. in Pure Math. 22, 1993, 53-68.

[I3] - Singularities of front mappings, in preparation.

[J] S. Janeczko, Generating families for images of Lagrangian submanifolds and open swallowtails, Math. Proc. Cambridge Philos. Soc. 100 (1986), 91-107.

[M1] J. N. Mather, Stability of $C^{\infty}$ mappings: V, Transversality, Adv. of Math. 4 (1970), 301-336.

[M2] - Stratifications and mappings, in: M. Peixoto (ed.), Dynamical Systems, Proceedings of Salvador Symposium on Dynamical Systems, Academic Press, 1973, 195-232.

[T] R. Thom, Singularities of differentiable mappings (notes by H. I. Levine), Bonn. Math. Schr. 1960; C. T. C. Wall (ed.), Proc. Singularities Sympos. Liverpool, Lecture Notes in Math. 192, Springer, 1971, 1-89.

[W] A. Weinstein, Lectures on Symplectic Manifolds, CBMS Regional Conf. Ser. in Math. 29, Amer. Math. Soc., 1977.

[Z] V. M. Zakalyukin, Generating ideals of Lagrangian varieties, in: Theory of Singularities and its Applications, V.I. Arnol'd (ed.), Adv. in Soviet Math. 1, Amer. Math. Soc., 1990, 201-210. 